\title{
Solar hydrogen-Lyman continuum observations with SOHO/SUMER
}

\author{
S. Parenti, P. Lemaire, and J.-C. Vial \\ Institut d'Astrophysique Spatiale, Bât. 121, Université Paris Sud-CNRS, 91405 Orsay, France \\ e-mail: Susanna.Parenti@medoc-ias.u-psud.fr
}

Received 13 May 2005 / Accepted 10 August 2005

\section{ABSTRACT}

In this work we analyze the emission of the H Lyman continuum (HI LyC) in the Quiet Sun (QS) and a solar prominence, in order to derive information on the temperature of the emitting plasma. We used the spectral atlas obtained with SOHO/SUMER detector A in 1999 (Parenti et al. 2005, A\&A, 443, 679). The high spectral resolution of this instrument allows a good selection of the continuum, free from emission lines. However, in the $\mathrm{HI} \mathrm{LyC}$ wavelength domain, the data suffer from large radiometric uncertainties, which lead us to use detector B as a reference. We obtained electron temperatures of $8281 \mathrm{~K} \pm 280 \mathrm{~K}$ and $7564 \mathrm{~K} \pm 230 \mathrm{~K}$ in two separate parts of the prominence.

Key words. Sun: prominences - Sun: UV radiation - Atlases

\section{Introduction}

The HI Lyman continuum (HI LyC) results from radiative recombination following ionization of neutral Hydrogen through collisional and radiative processes. In the case of prominences, radiative recombinations following photoionizations by the incident (strong) chromospheric emission is the primary process which essentially concerns the cool core of the structures (Heasley \& Milkey 1983). Prominences are very complex structures and, in order to understand their existence and their stability, it is extremely important to understand the mechanisms acting in their different layers. For this reason, the study of the HI LyC emission can give important information on such regions. Among the plasma parameters that can be obtained from the HI LyC diagnostic are the color temperature (given by the slope of the continuum) and brightness temperature (absolute intensity at the head of the continuum). Testing different models, Gouttebroze et al. (1993) show that the color temperature is very representative of the electron temperature for kinetic temperature $<15000 \mathrm{~K}$.

The first studies of the $\mathrm{HI} \mathrm{LyC}$ in prominences come from SKYLAB, OSO4, and OSO6 spectrometers operating in the seventies. Noyes et al. (1972) derived the color temperature of a prominence from the logarithmic derivative of the spectrum with respect to a quiet Sun (QS) continuum taken as a reference. Their results indicated a temperature of $6800 \mathrm{~K}$. Orrall \& Schmahl (1980) analyzed several spectra of prominences with the ATM Harvard EUV spectrometer. Using these data and two models for the prominence geometry, they derived several parameters, including color and brightness temperatures. Their results gave, on average, $7524 \mathrm{~K}$ for the color and $6316 \mathrm{~K}$ for the brightness temperatures. Schmahl et al. (1974), using the same instrument, found a lower average color temperature $(6600 \mathrm{~K})$, but with local variations between 5500 and $8000 \mathrm{~K}$.

The next observations of the HI LyC were made with SUMER on the SOHO mission. Quite large variations in the electron temperature (5000-15000 K) were found by Ofman et al. (1998) during the disappearance of a prominence; however, they used a quite narrow SUMER spectral window $(<30 \AA)$.

In this paper we investigate the properties of the H I Lyman continuum for the Quiet Sun (QS) and a prominence. The data we use here are part of a dataset, obtained during a MEDOC campaign in Orsay (FR), utilized by the authors to build a SUMER spectral atlas of prominence and QS. Parenti et al. (2004) (hereafter Paper I) describe the observations and the data reduction, while the atlas is presented in Parenti et al. (2005) (hereafter Paper II). The data were obtained in October 1999 by scanning the full wavelength range (800-1600 $\AA$ ) of SUMER detector A (Wilhelm et al. 1995, 1997; Lemaire et al. 1997). We observed the Sun center and a prominence in the south-west limb with the "reference spectra" sequence. The results presented in the atlas were obtained by averaging the spectra over two separate sections of the slit (named A_1 and A_2), while scanning the prominence, and by averaging the spectra over the entire slit while scanning the QS. Figure 1 shows an image of the prominence obtained by scanning the area in the H Lyman $\beta$ wavelength. Here we have marked the slit position (dashed line) and the two sections (solid white segments) where we averaged the spectra.

\section{Method}

The derivative of the logarithm of the continuum radiance versus the wavelength allows us to define the color temperature $\left(T_{\mathrm{c}}\right)$ of the emitting plasma. This temperature, for the QS 


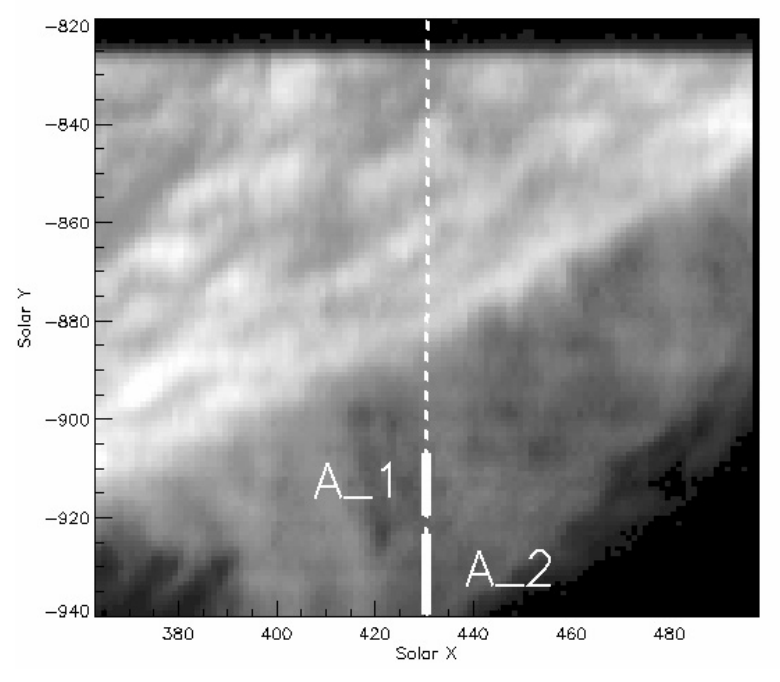

Fig. 1. Image in the $H$ Lyman $\beta$ line of the prominence obtained by rastering the area with SUMER slit 7. The position of the slit where the atlas spectrum was obtained is marked at the center of the raster by the white segment. A_1 and A_2 mark the two portions of the slit used for the present analysis.

and prominence cases, can indicate the local electron temperature $\left(T_{\mathrm{e}}\right)$ (Gouttebroze et al. 1993), as we show in the following.

For the observed radiance of QS and prominence plasmas, we can assume that the source function $\left(S_{\lambda}\right)$ varies linearly with the optical depth. In this case we can apply the EddingtonBarbier approximation (Noyes \& Kalkofen 1970) and write the radiance as $L_{\lambda}(\mu) \cong S_{\lambda}\left(\tau_{\lambda}=\mu\right)$, where $\mu$ is the cosine of the angle between the normal to the surface and the direction of the line of sight and $\tau$ the radial optical depth.

If we assume that the emitting region has a constant electron temperature, the source function can be represented by a black body $\left(B_{\lambda}\left(T_{\mathrm{e}}\right)\right)$ diluted by a factor $b$ (Brekke $\&$ Kjeldseth-Moe 1994):

$S_{\lambda}=\frac{B_{\lambda}\left(T_{\mathrm{e}}\right)}{b}$

where $S_{\lambda}$ is the source function and $b$ the departure coefficent of the ground state with respect to Local Thermodynamic Equilibrium (LTE). In this case, $T_{\mathrm{e}} \sim\left(T_{\mathrm{c}}\right)_{\mathrm{obs}}$ and $b$ is obtained by scaling $B_{\lambda}\left(T_{\mathrm{c}}\right)$ to reproduce the absolute values of the observed radiance.

From Eq. (1), applying the Wien approximation valid for short wavelengths and for $\mu=1$, we have

$L_{\lambda}=\frac{2 h c^{2}}{b \lambda^{5}} \exp \left(-\frac{h c}{\lambda k T_{\mathrm{e}}}\right)$

The parameters $b$ and $T_{\mathrm{e}}$ can be obtained by fitting the continuum radiance with Eq. (2).

\section{Data analysis}

For our analysis, we carefully selected the parts of the spectra which did not contain emission lines, either directly apparent in our data or observed in previous QS data. The radiances of

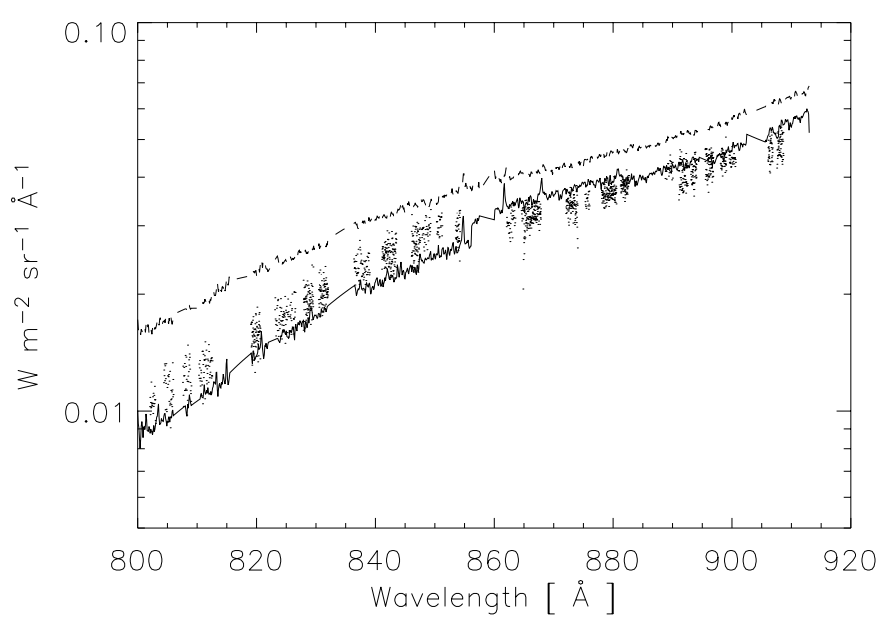

Fig. 2. QS HI LyC as a function of wavelength. The dots refer to our data. The dashed curve refers to detector A (1996 data) and the solid curve to detector B (1996-1998 data) from Lemaire et al. (2004).

the selected portions of spectra are plotted as function of wavelength in Fig. 4 for the QS (dots) and in Fig. 5 for the prominence (dots and crosses).

The study of the $\mathrm{H}$ continuum emission is not straightforward. The coefficient $b$, for example, is very sensitive to the radiometric calibration of the instrument used. In this part of the spectra, the radiometric calibration of detector A has large uncertainties due to the lack of reference emission lines below $800 \AA$ (Schühle et al. 2000). This results in an offset, not constant in wavelength, of the absolute radiances obtained from the two detectors (Fig. 2). Moreover, after the SOHO recovery, the uncertainty was raised from $15 \%$ to $33 \%$, and Lemaire et al. (2004) have raised this point, showing how detector B radiances are more compatible with SKYLAB data. A comparison with QS continuum calibrated profiles, of the same detector A, taken before and after the SOHO accident of 1998, shows small differences in the gradient and absolute values, as shown in Fig. 2.

In this figure we plot our calibrated data (dotted points), together with those of Lemaire et al. (2004), both for detector A (dashed curve, averaged over 1996) and detector B (solid curve, averaged over 1996-1998). On this plot we see the mentioned difference in the absolute values of the radiance between the two detectors. Moreover, the two datasets of detector A have different slopes.

To understand if this effect on detector A is due to a change in the QS data between the two periods or if it is instrumental, we plotted our uncalibrated data in Fig. 3 (dots) and compared them with the averaged QS uncalibrated detector A data of 1996 (Lemaire et al. 2004) (solid curve). In our case the number of counts/pixel is smaller, due to the change in time of the sensitivity of the detector and the use of a narrower slit. The difference between the slopes of the two curves, after the radiometric calibration has been applied, can be attributed to the uncertainty in the post-recovery calibration at such wavelengths.

To conclude the photometric issue, we believe that the most reliable radiometric calibration in this part of the spectra is 


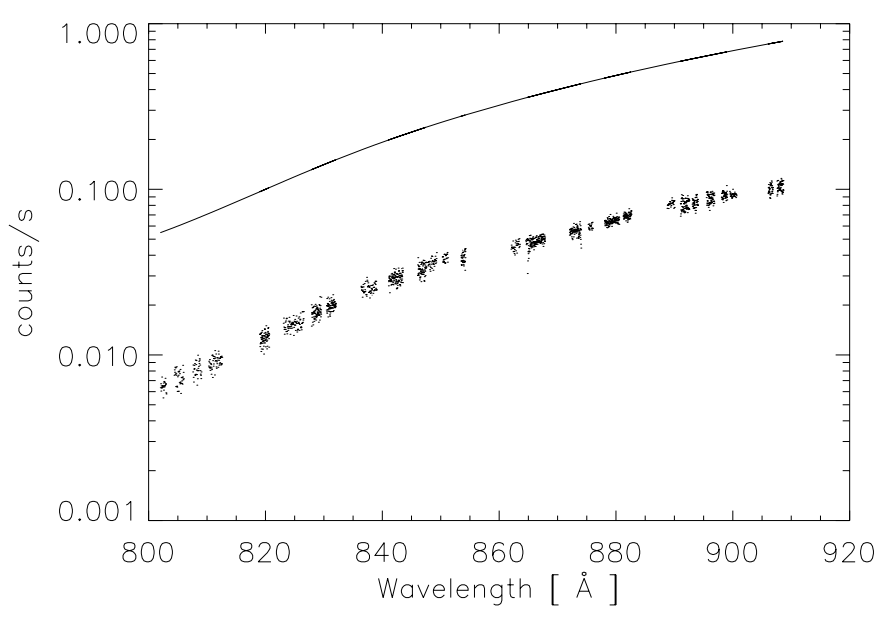

Fig. 3. QS HI LyC uncalibrated data as a function of wavelength. The solid curve refers to the Lemaire et al. (2004) detector A data. The dots refer to our data.

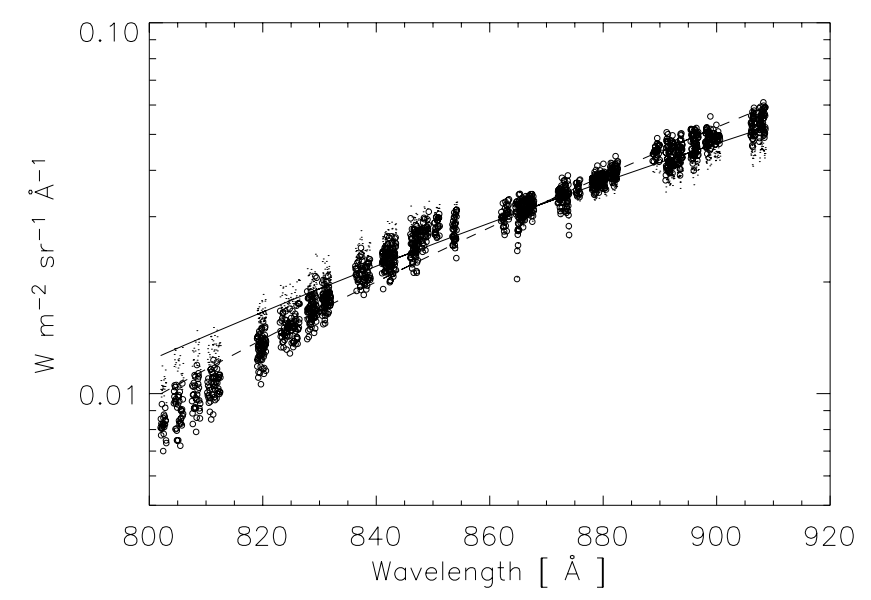

Fig. 4. Radiance of the H I LyC in the Quiet Sun as a function of wavelength. Open circles represent points corrected by the Lemaire et al. (2004) QS detector B data. The dots represent the uncorrected data. The curves represent fits to the two datasets (solid for the uncorrected data, dashed for the corrected).

that of detector B before 1998. For this reason, we decided to conduct the diagnostic investigation of our QS and prominence data, taking as reference the Lemaire et al. (2004) detector B QS data. For this detector, in this wavelength regime, the cited uncertainty is $36 \%$. In the following analysis we quantify the difference between Lemaire et al. (2004) detector B and our detector A radiometric QS data, in order to "correct" our data. This correction then solves the discrepancy in the absolute radiometry between the two detectors and the uncertainty of the calibration of detector A for post-recovery data.

Figure 4 shows (in dots) our QS data and the best fit determined with Eq. (2) (solid curve). We overplotted in empty dots our QS data corrected for the Lemaire et al. (2004) curve together with its best fit (dashed curve). We clearly see that the correction leads to a steepening of the continuum.

Figure 5 shows in dots the original prominence continuum. In Paper I we pointed out that these data show evidence of temporal variations due, probably, to the non-simultaneity of the observations. This effect is particularly important in A_1
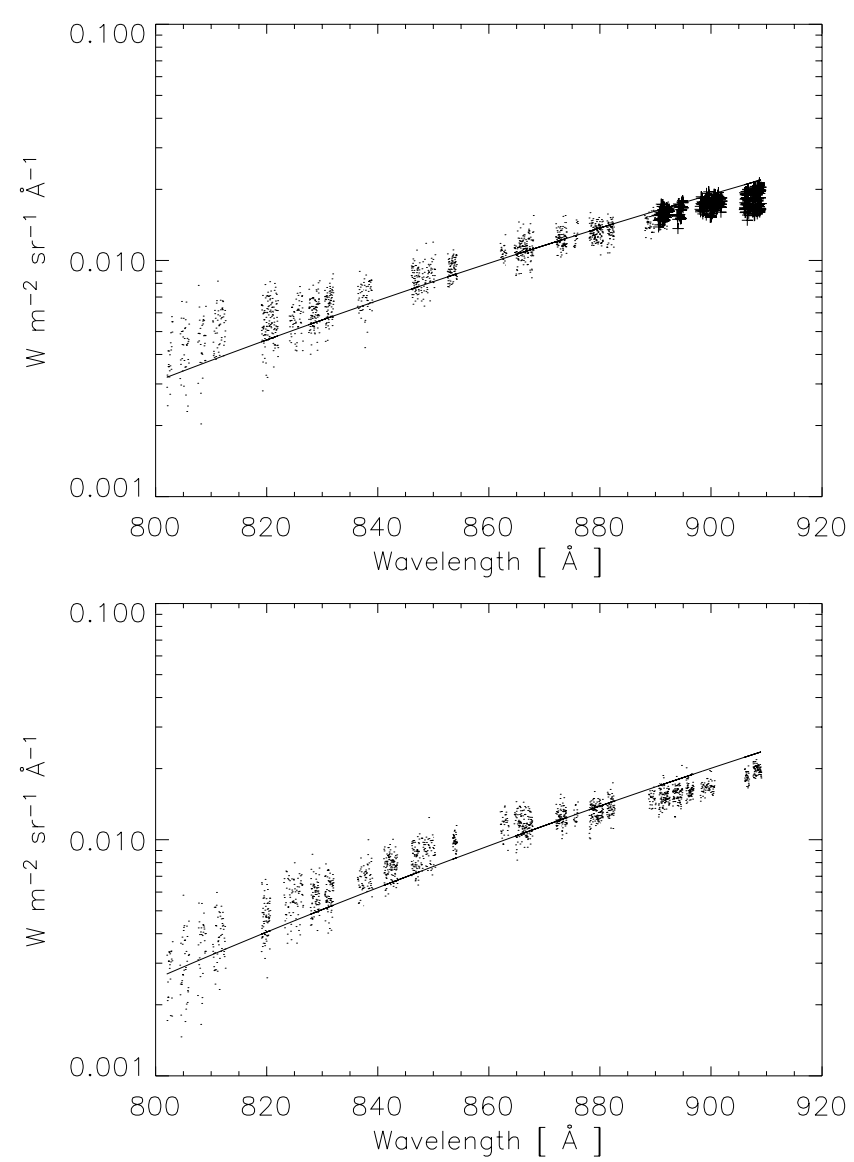

Fig. 5. Radiance of the H I LyC in prominence A_1 (Top) and A_2 (Bottom) as a function of wavelength. The crosses in the top plot indicate the data corrected for temporal variations. The solid curve in each plot represents the fit to the corrected data by applying the results derived from the QS (Fig. 4).

at the time we were observing the H Lyman head region. We estimated that the variation of the continuum radiance was about $26 \%$. For the present analysis, this variation involves only a small part of the data $(\lambda>890 \AA)$.

Under the assumption that the temporal variation does not alter the slope of the continuum radiance, spectra belonging to the same wavelength range, but taken in different exposures, should have similar intensity gradients. For this reason we corrected the observed temporal variations by imposing the same radiance at such wavelengths. This was possible by taking advantage of the spectral superposition of two successive windows. The data corrected in this way were plotted in Fig. 5 (top) as crosses.

The next step in the analysis then is to correct all the data points by the amount estimated from the QS case. The fit with Eq. (2) to the corrected prominence data is overplotted in Fig. 5 (Top), while the results from the fitting in terms of electron temperature and $b$ are given in Table 1 . To verify the effect of our correction on the A_1 data at the head of HI LyC, we made the same analysis discarding these parts of the data. The results from the fitting gives a variation less than $1 \%$ on the temperatures and about $18 \%$ on the factor $b$. 
Table 1. Results from the present analysis. The first column reports the radiance at the $\mathrm{HI}$ LyC head. Its uncertainty is $33 \%$ given by the radiometric calibration. The brightness temperature at the Lyman head and its error are given in the second and third columns. The other columns give the results from the fit of the $\mathrm{HI} \mathrm{LyC}$ in the QS and prominence using Eq. (2). The fits were for the data corrected to the QS of Lemaire et al. (2004). For each parameter, we provide its standard deviation, representative of the deviation of the data from Eq. (2), where only one value for $T_{\mathrm{e}}$ and $b$ was assumed. Temperatures are in Kelvin, and radiances in $\mathrm{mW} \mathrm{m} \mathrm{m}^{2} \mathrm{sr}^{-1} \AA^{-1}$.

\begin{tabular}{lccccccc}
\hline \hline & $L_{\mathrm{o}}$ & $T_{\mathrm{B}}$ & $\sigma_{T_{\mathrm{B}}}$ & $T$ & $\sigma_{T}$ & $b$ & $\sigma_{\mathrm{b}}$ \\
\hline QS (corrected) & 62.2 & 6536 & 105 & 8753 & 10 & 452 & 11 \\
Prom. A_1 & 23.0 & 6277 & 96 & 8281 & 281 & 438 & 301 \\
Prom. A_2 & 24.8 & 6296 & 97 & 7564 & 231 & 67 & 44 \\
\hline
\end{tabular}

Figure 5 (bottom) shows the results of the same analysis for the prominence A_2. These data lead to a slightly steeper continuum than for A_1 and, consequently, a lower temperature (Table 1).

Table 1 also reports the brightness temperature $\left(T_{\mathrm{B}}\right)$ calculated at the HI LyC head. This value is similar for the two prominence sections, while slightly higher for the QS.

\section{Discussion}

We have presented here analysis of the HI LyC emission for the quiet Sun and a prominence observed in 1999. The use of SUMER data for the analysis is a big improvement over previous studies, because a high spectral resolution is essential in the selection of line-free spectral windows.

Comparing our QS data with observations prior to the SOHO recovery from both SUMER detectors, has revealed disagreement between the different datasets. We established that an important uncertainty exists in the radiometry calibration of detector A at the shorter wavelengths, post-recovery. We determined a correction to apply to these data in order to properly diagnose the observed plasma.

The QS brightness temperature is compatible with the values obtained by Wilhelm \& Kalkofen (2003) using SUMER data from the same period. Moreover, our analysis indicates a lower $T_{\mathrm{B}}$ in the prominence than in the QS.

The electron temperatures we obtained for the prominence, using the corrected data, indicate that the area A_2 is cooler than A_1. These two sections have the same brightness temperature, but the continuum gradient is slightly different. In Paper I we already pointed out the different properties of the two prominence sections and the fact that A_1 seems to be more inhomogeneous, with a large transition region and coronal contributions.

Comparing the prominence results with previous observations, we found that our temperatures are higher than the $6800 \mathrm{~K}( \pm 500 \mathrm{~K})$ derived from OSO 4 and OSO6 data (Noyes et al. 1972). However, their results are scaled to a reference QS temperature of $8400 \mathrm{~K}$, which is slightly lower than our QS value.

On the other hand, we found that our color and brightness temperatures agree well with the average values of
$7524 \mathrm{~K} \pm 739 \mathrm{~K}$ for the color and $6316 \mathrm{~K} \pm 75 \mathrm{~K}$ for the brightness temperature obtained by Orrall \& Schmahl (1980). In particular, our prominence has very similar parameters to prominence No. 8 of these authors $\left(L_{\mathrm{o}}=24.1, T_{\mathrm{c}}=7153 \pm 772 \mathrm{~K}\right.$, $\left.T_{\mathrm{B}}=6290 \mathrm{~K}\right)$. From their analysis, the optical depth $\tau_{912}$ is 6.5 for a prominence with a "slab" geometry and 12.8 for a "thread" geometry. Using an isothermal slab model, Heasley $\&$ Milkey (1983) predicted much lower values for $\tau_{912}$ for the same range of $L_{0}$. These different results show how the assumed geometry affects the results, which indicates the necessity of imposing more observational constraints on the models.

We also compared our results with those predicted by the most recent prominence models of Gouttebroze et al. (1993). Selecting the model which assumes an electron temperature of $8000 \mathrm{~K}$, similar to what we obtained with the present analysis, these authors predict a HI LyC head intensity of about $21 \mathrm{~mW} \mathrm{~m}^{2} \mathrm{sr}^{-1} \AA^{-1}$, which agrees well with our values. These authors also show that for temperature less than $10000 \mathrm{~K}$, this intensity depends only on the incident radiation. This can be an indication that the principal ionizing process is the absorption of solar chromospheric radiation. In this context, we calculated the dilution factor at the average position where we observed the prominence (26 Mm off-limb) and found 0.37 , a value comparable with the ratio of the measured $\mathrm{HI}$ LyC head intensity of the prominence to the QS (0.38). This confirms that the role of solar chromospheric radiation (in this case photoionization followed by radiative recombination) is dominant in the $\mathrm{HI} \mathrm{LyC}$ prominence emission (see also Fig. 16 of Heinzel et al. 1994).

Our $b$ values (438 and 67) are also consistent with Gouttebroze et al. (1993), who found about 200. In contrast, earlier works obtained values of $b<14$ (Schmahl et al. 1974; Noyes et al. 1972; Kanno et al. 1981). The present analysis gives quite high uncertainty on this parameter. There are three main sources for this. First, over a small wavelength range our data show quite a high dispersion from the average intensity value. The second aspect is the uncertainty on the radiometric calibration, which is assumed to be constant over our wavelength range (33\%). Local variations of absolute values cannot be evaluated well, because the absolute calibration can be obtained only for a few wavelengths. Finally, we made an assumption on the physical condition of the observed plasma, that is, unique $b$ and $T_{\mathrm{e}}$ values for the full wavelength range.

In summary, the high resolution spectra obtained with SUMER in the QS and in the prominence have allowed us to derive the electron temperature of the emitting plasmas. In two parts of the prominence, the temperature is in the range of $7500 \mathrm{~K}$ to $8300 \mathrm{~K}$ with errors bars $(\sim 250 \mathrm{~K})$ lower than in previous studies. The investigation of observed and modelled Lyman lines, constrained by the above empirical temperature, should yield considerable new information on prominence thermodynamics.

On the basis of the prominence atlas, we plan to further investigate the hydrogen emission in a forthcoming paper.

Acknowledgements. The authors wish to thank the referee for careful revision of the work. S.P. would like to thank K. Wilhelm for his comments on the manuscript and the MEDOC team for providing the data. SUMER is financially supported by DLR, CNES, NASA, and 
the ESA PRODEX program (Swiss contribution). SOHO is a mission of international cooperation between ESA and NASA. SP was supported through the TOSTISP European network (contract \# HPRNCT 200100310).

\section{References}

Brekke, P., \& Kjeldseth-Moe, O. 1994, Sol. Phys., 150, 19

Gouttebroze, P., Heinzel, P., \& Vial, J. C. 1993, A\&AS, 99, 513

Heasley, J. N., \& Milkey, R. W. 1983, ApJ, 268, 398

Heinzel, P., Gouttebroze, P., \& Vial, J.-C. 1994, A\&A, 292, 656

Kanno, M., Withbroe, G. L., \& Noyes, R. W. 1981, Sol. Phys., 69, 313

Lemaire, P., Wilhelm, K., Curdt, W., et al. 1997, Sol. Phys., 170, 105

Lemaire, P., Gouttebroze, P., Vial, J.-C., et al. 2004, A\&A, 418, 737
Noyes, R. W., Dupree, A. K., Huber, M. C. E., et al. 1972, ApJ, 178, 515

Noyes, R. W., \& Kalkofen, W. 1970, Sol. Phys., 15, 120

Ofman, L., Kucera, T. A., Mouradian, Z., \& Poland, A. I. 1998, Sol. Phys., 183, 97

Orrall, F. Q., \& Schmahl, E. J. 1980, ApJ, 240, 908

Parenti, S., Vial, J.-C., \& Lemaire, P. 2004, Sol. Phys., 220, 61

Parenti, S., Vial, J.-C., \& Lemaire, P. 2005, A\&A, 443, 679

Schühle, U., Curdt, W., Hollandt, J., et al. 2000, Appl. Opt., 39, 418

Schmahl, E. J., Foukal, P. V., Noyes, R. W., et al. 1974, Sol. Phys., 39, 337

Wilhelm, K., \& Kalkofen, W. 2003, A\&A, 408, 1137

Wilhelm, K., Curdt, W., Marsch, E., et al. 1995, Sol. Phys., 162, 189

Wilhelm, K., Lemaire, P., Curdt, W., et al. 1997, Sol. Phys., 170, 75 